\title{
Evaluation of Academic Competitiveness Based on Scientific Research Papers
}

\author{
Chen Huaxiong ${ }^{1}$, Wang Jian ${ }^{2, *}$ \\ ${ }^{1}$ Center of Science and Technology Evaluation, Ministry of Science and Technology, Beijing, China \\ ${ }^{2}$ School of Mechanical Engineering and Automation, Beihang University, Beijing, China
}

Email address:

chenhuaxiong@ncste.org (Chen Huaxiong),wj279107698@163.com (Wang Jian)

*Corresponding author

\section{To cite this article:}

Chen Huaxiong, Wang Jian. Evaluation of Academic Competitiveness Based on Scientific Research Papers. International Journal of Science, Technology and Society. Vol. 6, No. 5, 2018, pp. 78-87. doi: 10.11648/j.ijsts.20180605.12

Received: October 9, 2018; Accepted: November 13, 2018; Published: December 20, 2018

\begin{abstract}
To evaluate the capabilities of academic and scientific research and to support the optimal allocation of scientific research resources as well as the strategic decisions pertaining to science and technology policy, a scientific competitiveness evaluation model is established based on the value of scientific research papers. Using bibliometric and innovation network analysis, an evaluation index is constructed, including quantitative, influence, and frontier of scientific research innovation indicators. The quantitative indicator is based on the citation's number of scientific research papers. By using between centrality, the influence indicator is calculated. Considering the hot spots and influence of scientific research papers, the frontier indicator is calculated by between centrality and burst detective algorithms. A comprehensive evaluation of academic competitiveness was completed in the scientific research field using the Technique for Order Preference by Similarity to Ideal Solution (TOPSIS) algorithm. Empirical research was conducted on the academic competitiveness of countries in the field of graphene. Through the analysis of the scientific competitiveness of major countries, the results showed that the published papers and citations of China ranked in the world. The United Kingdom ranked first in the frontier, and Germany ranked first in influence. From the comprehensive evaluation perspective, the United States achieved good results in quantity, centrality, and hot spots, and ranked first in the world. Germany, Britain, China, and Spain were ranked from second to fifth place, respectively. The results of each index and comprehensive ranking evaluation of graphene were consistent with expert surveys.
\end{abstract}

Keywords: Academic Evaluation, Competitiveness Evaluation, Evaluation Model Index System, Comprehensive Evaluation

\section{Introduction}

With the development of technology and the global economy, competition among countries has moved forward to the front of the innovation chain. Countries around the world have invested huge humanpower and material resources to promote research and development. The innovation ability and scientific research level of a country's basic research has become an indication of a nation's level of technology and national defense strength as well as an important symbol of overall national strength [1]. At present, the development of science and technology in China has entered "the coexistence stage" including a leading, reaching stage and following stages [2]. Although some scientific research fields are leading globally, some scientific research fields and innovative subjects have entered the "no man's land" stage of research, in which there are no leaders, no rules, no followers. The promotion of innovation ability and the scientific research level in China has become a difficult problem to be solved [3].

China has promulgated a series of incentive policies, including the "Outline of the National Strategy of Innovation-Driven Development," which is designed to strengthen the original innovation, the supply of the source, and the research frontier of the national strategic demand as well as research and development of subversive technology. To evaluate current scientific and technological innovation in China, it is necessary to establish a macro-evaluation method and an index system for the innovation ability and scientific research level. It is of great significance to be able to optimize the allocation of scientific resources in China and to anticipate the layout of disruptive technology and new industries. 
Scholars have conducted a lot of research on the evaluation of academic competitiveness at home and abroad. Different types of evaluation systems and methods have been constructed. Peer evaluation and bibliometrics method are widely used in academic competitiveness evaluation. The evaluation of scientists and the achievements of scientific research usually are based on peer review. Although some metrics are used for evaluation, including award-winning research, number of published papers, number of quotations, and h-index, no method is available for the comprehensive evaluation of a scientific research field [4]. Scholars have noted that the assessment of the field of basic research should follow several principles: (1) the aim of the research is to guide and promote the realization of national scientific goals and to establish a scientific research system; (2) the evaluation process needs to combine national scientific goals with high standards and quality; and (3) the quantitative indicators of basic research should include the quantity and quality of the treatises [5].

As noted, peer evaluation is a widely used evaluation method in academic evaluation of the scientific research field. Different scholars have different views on the specific content of peer evaluation involving scientific evaluation. Some scholars believe that the emphasis of peer review lies in the qualitative evaluation of the research frontier of basic research projects, operation of equipment, contribution to the society, and international status [6]. Other scholars believe that the evaluation of basic research should be compared with project participants, use of funds, papers, awards, patents, industrialization, follow-up support and training of talent. When evaluating innovation and talent, the training of their research ability should be the focus [7]. Yet other scholars believe that basic research performance evaluation is related to the self-construction and sustainable development of the subject, including talent structure and team spirit.

Bibliometrics is a method used to evaluate performance by means of statistical analysis of papers or patent documents, which share research results [4]. As early as 1970s, industrial countries began to evaluate and predict academic research innovation through bibliometric analysis [9]. The main measurement objects of bibliometrics include published papers, monographs, information collected by retrieval systems, the citation of documents, the application of patents, and the acceptance of documents. There are many studies on the evaluation of literature, quality of periodicals, and influence of scientists based on quantitative evaluation, including the number of references, which is the influence factor of academic journals [10-12], and scientists' h-index. Some scholars have introduced the concept of "centrality" in the network to this type of evaluation, which indicates the importance and influence of a work of literature, a writer, or an organization relative to the scientific community. In 1998, Freeman first proposed the concept of centrality in social network analysis [13-17], which was translated into a literature co-citation network to represent the control of nodes later represented in a paper in an entire network. In CiteSpace, a document analysis visualization tool developed by Professor Chen Chaomei, intermediary centrality has become one of the most important indicators of scientific research evaluation to measure scientific measurement units, including authors and quotations.

Scholars also have studied the evaluation of academic innovation in specific scientific research fields. Zhang Jing [19] from Northwest Agriculture and Forestry University constructed an evaluation index system of technological innovation ability in agriculture according to two aspects: (1) knowledge creation ability of agricultural science and (2) technology innovation, knowledge application ability of scientific, and technological innovation. Wang Zeyu [20] used an analytic hierarchy process and comprehensive index method to evaluate the developing marine science and technology innovation ability and marine economic development in the coastal areas of China. Gao Feng [21] evaluated the scientific and technological innovation in the field of civil aviation by using four indexes, namely, basic level of science and technology, investment of scientific and technological activities, achievement of scientific and technological activities, and transformation ability of science and technology. Zhou Jian [22] proposed an evaluation index and method to evaluate the manufacturing industry and related enterprises' scientific and technological innovation.

At present, the evaluation of the scientific research field focuses on the evaluation of achievement results, including papers, monographs, conferences, and patents. Very few indicators, however, reflect the long-term effects [23]. The performance evaluation of the scientific research field conducted abroad focuses more heavily on the quality and social influence of research. For example, the British evaluation system of scientific research level suggests that scientific research evaluation not only is limited to the number of scientific research papers but also should pay more attention to the quality of scientific research and the contribution of scientific research to the economy and society, including the evaluation of scientific research influence.

\section{The Establishment of the Evaluation Model of Academic Competitiveness}

The evaluation of academic competitiveness in the scientific research field is based on the measurement method of academic papers, which is to extract quantitative indicators, including the quantity, influence, and frontier of scientific research innovation. On the basis of a comprehensive evaluation algorithm, the relative strength of the academic ability and level of scientific research in a particular field was determined and the relative competitiveness of different countries in specific areas was identified. Many factors affect academic competitiveness in a scientific research field, including investment, achievement, organization, implementation, and research environment. During final analysis, the academic competitiveness of a scientific research field was determined according to the achievements and the value of the achievement in the relevant scientific field. 
Generally, the achievements of scientific research include monographs, periodical papers, and conference papers. The value of creation mainly includes the discovery of the law of science, the creation of basic theory, and the dissemination of professional knowledge. The results are applied by the peers and the public to produce economic and social values. The

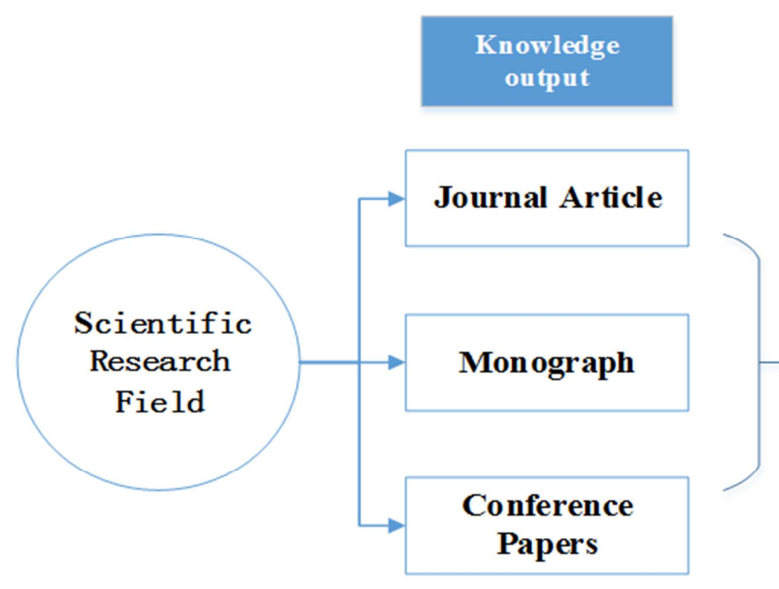

value created by scientific research has become the foundation and source of the value of scientific and technological innovation. From the perspective of evaluation approaches, academic competitiveness was characterized according to a quantitative indicator, influence indicator, and frontier of scientific research innovation, as shown in Figure 1.

Figure 1. Evaluation model of academic competitiveness in the scientific research field.

The evaluation of academic competitiveness in key scientific research fields assessed the basic scientific research achievements in this field. It reasonably reflected the ability and level of academic research in this key scientific field through the analysis and evaluation of published papers, monographs, and reports. In the existing research, the quantitative indicator and influence indicator generally are regarded as important evaluation indexes. The quantitative indicator includes the number of papers published as well as the number of citations. The influence indicator generally is obtained by expert investigation or through quantitative calculation. By using the software and methods of literature analysis, the frontier index data of academic research was extracted and the relationship between relevant indicators and academic frontiers was verified according to peer expert interviews. A strong correlation existed between the duration of hot spots and the technological frontier. Technological frontier represents new technological directions and future industry developments that play leading roles in the economy and society.

\section{Evaluation Indicator and Method of Academic Competitiveness}

On the basis of the evaluation model of academic competitiveness, our evaluation of the competitiveness of key scientific research fields included three primary aspects: the quantitative indicator, the influence indicator, and the hot spot and frontier indicator of scientific research innovation, which was objectively obtained according to literature statistics and measurement tools. Finally, the Technique for Order Preference by Similarity to Ideal Solution (TOPSIS) algorithm was applied to rank scientific competitiveness in different countries and regions.

\subsection{Evaluating Indicator of Academic Competitiveness}

\subsubsection{Quantitative Indicator of Scientific Research Innovation}

The quantitative indicator of scientific research innovation includes the number of publications and the number of citations. This indicator has a great number of citations under the hyper-thesis of using a huge number of publications to evaluate a country's academic competitiveness. Past studies found that the number of publications and citations can be regarded as the same indicator. In national rankings, the results of the total number of publications and citations are always consistent, which is different from the evaluation of the scientific research of individuals. In the evaluation of specific scientists or papers, the number of citations usually characterizes quality, which means that the greater the number of citations, the higher level of quality.

Studies have shown that total citations of scientific papers can more effectively reflect the number of academic innovations. When two papers on the same topic were cited by a third paper at the same time, we identified the citing paper as belonging to the same research topic, and the citation of the cited paper was considered to be a valid citation. If the frequency of total citations was higher, this meant that the paper had been applied more times by the peer. By comparing the results of repeated calculations with the results of an expert survey, the total citations more precisely reflected the value of scientific research, which more accurately represented the number of academic innovations in the scientific research field.

\subsubsection{Influence Indicator of Scientific Research Innovation}

The influence indicator of scientific research and academic innovation refers to the influence of scientific research achievements for other countries and regions in a certain 
scientific research field. This paper introduced centrality indicators to describe the influence of academic innovation. Centrality indicators were used to analyze the citation network. These centrality indicators reflected the degree of attention an academic paper received in this field. If the centrality indicators were higher, the paper was in a central position in the quoted network and was more widely recognized by peers from different countries and regions.

Freemen first proposed centrality in social network analysis, which has been developed as a key aspect of network analysis. Centrality refers to the probability that the shortest path between any two points in a network passes through a certain node. In general, the more an actor is in the center of a network, the greater that actor's influence. In social network analysis, the importance of nodes is called centrality. The importance of nodes is equivalent to the connection of nodes to other nodes that make it more significant [27]. The more neighbors a node has, the greater influence it will have.

In the analysis of the citation network, Between centrality was used to represent the influence of academic papers in the network. This variable indicated that the shorter the path through a node, the more important that node. Between centrality indicates the control of the network flow along the shortest path in the network. The number of nodes is defined as follows:

$$
B C(i)=\sum_{i \neq s, i \neq t, i \neq t} \frac{g_{s t}^{i}}{g_{s t}}
$$

In bibliometric statistics, the Between centrality of a technology or thesis indicates the extent and scope of recognition by different people, which reflects the influence of academic innovation.

\subsubsection{Hot Spots and Frontier Indicator of Scientific Research Innovation}

The indicator for hot spots of scientific research innovation refers to a hot topic of research that has received extensive attention from experts and researchers in an area, which includes in-depth exploration and analysis and effective application. The indicator for hot spots of scientific research innovation represents a mainstream research direction. The frontier of academic innovation refers to the most advanced, latest, and most promising research topics in a scientific research field. It represents the difficulties, hot spots, and trends in scientific development, and is forward-looking. The direction of the development of the discipline is led by a few scientific research frontiers, and the development of the discipline can be promoted significantly by most of the research results.

With the development of the network and the increasingly frequent flow of academic resources, scholars have proposed a burst detective algorithm to identify emerging research frontiers. The burst detective algorithm is suitable for citation analysis of multiword terms and time series. Burst detection has several common meanings, such as sudden change and sudden increase. It is the value of a variable that varies greatly in a short time. Through this burst value, the technology that is focused in a certain field can be extracted quickly in a short time. Additionally, the extracted technology can be namely as "hot" technology. Hot technology may trigger new directions and turning points, which is essential for research in a field. A result's hot spots indicate that a given technology has great potential. As time develops, some hot spots will disappear and other hot spots may generate new hot spots.

In this paper, the Sigma index was used to characterize the frontier of science. The Sigma index is a comprehensive algorithm of centrality and burst values. It represents a continuous hot spot of a topic or research achievement. When a technique has been widely cited by experts and scholars in the same field for a long time and generated a new research results, it produces new research results. As more research has been carried out based on this technology, a new technical direction will be derived, which will become the frontier in this field. The Sigma indicator algorithm is as follows:

$$
\text { Sigma }=(\text { centrality }+1)^{\text {burstness }}
$$

In some fields, theoretical research and disruptive technical discoveries have a long process of recognition. After long-term monitoring and verification, the research will be widely cited as original results by scholars from around the world, and the quantitative indicator values will be quite high. Some emerging studies carried out on this basis become new high hot spots. This does not mean, however, that the centrality and the Sigma index will be relatively high. Through a large number of comparative studies, the Sigma index was found to be better than the burst value when characterizing the frontier of technology research.

\subsection{Evaluation Methods and Software}

\subsubsection{A method of Academic Competitiveness}

Academic competitiveness is the performance of comprehensive strength. The assessment of competitiveness in a scientific field uses evaluation methods and consistent software to obtain index values. On the basis of these index values, a comprehensive evaluation of various indicators was conducted to form a definite evaluation conclusion. In this paper, a method for academic competitiveness assessment was established using CiteSpace software, TOPSIS algorithm, and expert verification.

The method is as follows:

Step 1: To evaluate academic papers in a scientific research, extract the key words [A1, A2, Aa] of the academic papers.

Step 2: According to key words in these papers, these papers retrieved similar papers related to the academic papers in the database and the network citation's data in the field were obtained.

Step 3: According to the network citation's data in the field of evaluation in step 2, select papers according to country and region. Calculate the quantitative indicator $N$, the influence indicators $B C$ of scientific research innovation, and the hot spots and the frontier Sigma indicator values of scientific research innovation in scientific research in the set time 
interval $T$ were calculated.

Step 4: According to the indicator value of the quantitative indicator $N$, the influence indicators $B C$, and the hot spots and the frontier Sigma indicator values of the research field paper in step 3, the proximity index $C_{i}^{*}$ is calculated and the papers based on the TOPSIS algorithm is ranked.

Step 5: According to the proximity index $C_{i}^{*}$ and rank of the technologies described in step 4, the academic competitiveness of different countries and regions is obtained.

\subsubsection{TOPSIS Algorithm}

TOPSIS algorithm is a common method for multiple target decision analysis in system engineering, which can be used to comprehensively rank evaluation objects with multiple indicators. On the basis of the normalized original data matrix, the optimal scheme and the worst scheme in the finite scheme were found. The distance between the evaluation object with the optimal scheme and the worst scheme was calculated, and the relative degree of closeness between each evaluation object and the optimal plan were obtained, which formed the basis for evaluating merits and demerits.

The steps of TOPSIS algorithm follow:

Step 1: Calculate the Normative decision matrix $Z$ by a vector specification-based approach:

$$
z_{i j}=y_{i j} / \sqrt{\sum_{i=1}^{m} y_{i j}^{2}} .
$$

Step 2: Construct the weighted standard array X:

$$
x_{i j}=w_{j} \cdot z_{i j}
$$

Step 3: Determine the ideal and negative ideal solutions: Ideal solution

$$
x_{j}^{*}=\left\{\begin{array}{l}
\max _{i} x_{i j} \\
\min _{i} x_{i j}
\end{array}\right.
$$

Negative ideal solution

$$
x_{j}^{0}=\left\{\begin{array}{l}
\min _{i} x_{i j} \\
\max _{i} x_{i j}
\end{array}\right.
$$

Step 4: Calculate the distance between the ideal solution and the negative ideal solution:

The distance to the ideal solution

$$
d_{i}^{*}=\sqrt{\sum_{j=1}^{n}\left(x_{i j}-x_{j}^{*}\right)^{2}}
$$

The distance to the negative ideal solution

$$
d_{i}^{0}=\sqrt{\sum_{j=1}^{n}\left(x_{i j}-x_{j}^{0}\right)^{2}}
$$

Step 5: Calculate the closeness of each solution to the ideal solution:

$$
C_{i}^{*}=d_{i}^{0} /\left(d_{i}^{0}+d_{i}^{*}\right)
$$

Step 6: Aarrange the order of strengths and weaknesses in descending order..

\subsubsection{Data Acquisition}

By using the Web of Science database, CiteSpace software, and information visualization software, the citation information was collected for evaluation. The citations and the data of

\begin{tabular}{|c|c|c|c|}
\hline TOPIC & Topic definition & Range & Analysis content \\
\hline $\begin{array}{l}\text { Analysis of national } \\
\text { cooperation }\end{array}$ & $\begin{array}{l}\text { the state's contribution to the } \\
\text { field of research }\end{array}$ & Country & $\begin{array}{l}\text { The number of papers issued by the state (region), the total cited frequency, the hot } \\
\text { spot, and the centrality analysis.. }\end{array}$ \\
\hline $\begin{array}{l}\text { Analysis of } \\
\text { institutional } \\
\text { cooperation }\end{array}$ & $\begin{array}{l}\text { the contribution of institutions } \\
\text { to the field of research }\end{array}$ & Institution & $\begin{array}{l}\text { List of institutions with a high volume of publications; major research institutions } \\
\text { and regional distribution analysis; major research institutions issuing volume and } \\
\text { research priorities; and inter interagency cooperation analysis. }\end{array}$ \\
\hline $\begin{array}{l}\text { Co-citation analysis } \\
\text { of the author }\end{array}$ & $\begin{array}{l}\text { the academic relevance } \\
\text { between the authors }\end{array}$ & Cited Author & $\begin{array}{l}\text { The main topic names and representatives are listed by the clustering of the co } \\
\text { cited network. The names of the authors, the cited frequency and the centrality are } \\
\text { enumerated according to the frequency of citation. }\end{array}$ \\
\hline $\begin{array}{l}\text { Subject field } \\
\text { analysis }\end{array}$ & $\begin{array}{l}\text { the composition of the subject } \\
\text { in the field of research }\end{array}$ & Category & $\begin{array}{l}\text { The research field is divided into disciplines; the representative figures and } \\
\text { representative works in different disciplines; the key nodes are used to analyze the } \\
\text { correlation among disciplines. }\end{array}$ \\
\hline $\begin{array}{l}\text { Literature co } \\
\text { citation analysis }\end{array}$ & $\begin{array}{l}\text { the topic relevance between } \\
\text { the literature }\end{array}$ & Cited Reference & $\begin{array}{l}\text { According to the cited frequency, the author, title, source journal, year of } \\
\text { publication and citation frequency are listed. }\end{array}$ \\
\hline $\begin{array}{l}\text { The analysis of } \\
\text { thematic words }\end{array}$ & $\begin{array}{l}\text { the topics in the field of } \\
\text { research }\end{array}$ & Term & $\begin{array}{l}\text { Thematic words are clustered together in the network, analyzing and studying } \\
\text { thematic fields, lists of high-frequency keywords, and analysis of thematic } \\
\text { connotation and representative works. }\end{array}$ \\
\hline
\end{tabular}
knowledge were transformed into visual representations using software calculation. A development trend of the technique in a certain period was presented and the evolution and change course of several research frontiers were established. The analysis of CiteSpace is shown in Table 1.

Table 1. Analysis of CiteSpace. 


\subsubsection{Expert Consultation and Evaluation}

We invited experts in the field of technology to conduct investigations and interviews and to consult on issues such as domain classification, key technologies, and evaluation indicators. The experts judged the rationality of the ranking evaluation, and experts' suggestions on academic innovation and technological innovation in the field helped optimize the evaluation results.

\section{Empirical Evaluation of the Scientific Competitiveness of Graphene}

\subsection{Overview of the Academic Research of Graphene}

We used graphene as an example to evaluate scientific competitiveness of major countries in the world and to conduct empirical research on the methods and systems used for evaluating academic competitiveness. Seventy countries and regions published research in the field of graphene, including China; the United States; South Korea; Japan; Germany; India; Singapore; the United Kingdom; France; Spain; and Taiwan, China. By the end of November 2017, 126,752 scientific and technical research articles with the subjective word "graphene" were identified in the Web of Science database. From the perspective of annual distribution, research documents on graphene were first published in 1991. An inflection point was reached in 2005. Since 2013, the number of graphene papers has been increasing at a rate of more than 10,000 every year. Global attention on graphene has continued to increase and has become a hot spot in global research. The number of graphene papers published from 1991 to 2017 is shown in Figure 2. The quantity of graphene papers published in major countries is shown in Figure 3. The funding and the primary support mechanism for published papers are given in Table 2.

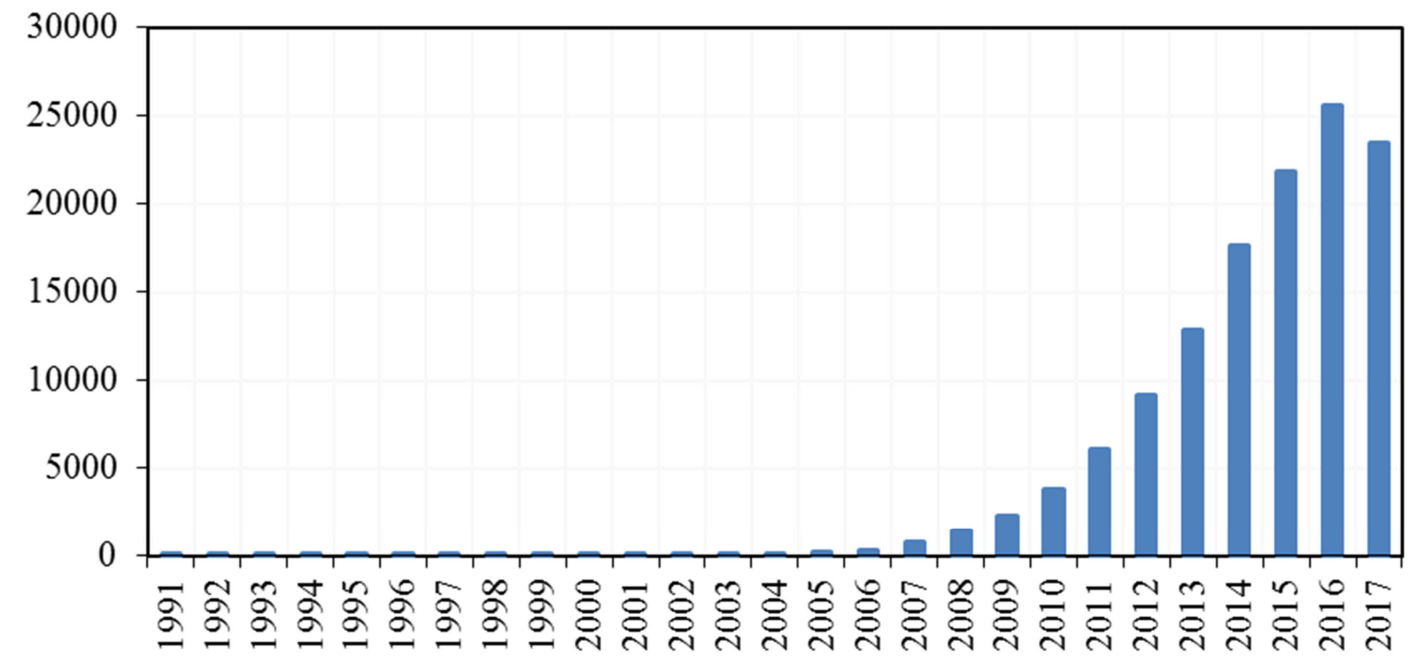

Figure 2. Number of graphene papers over the past 26 years.

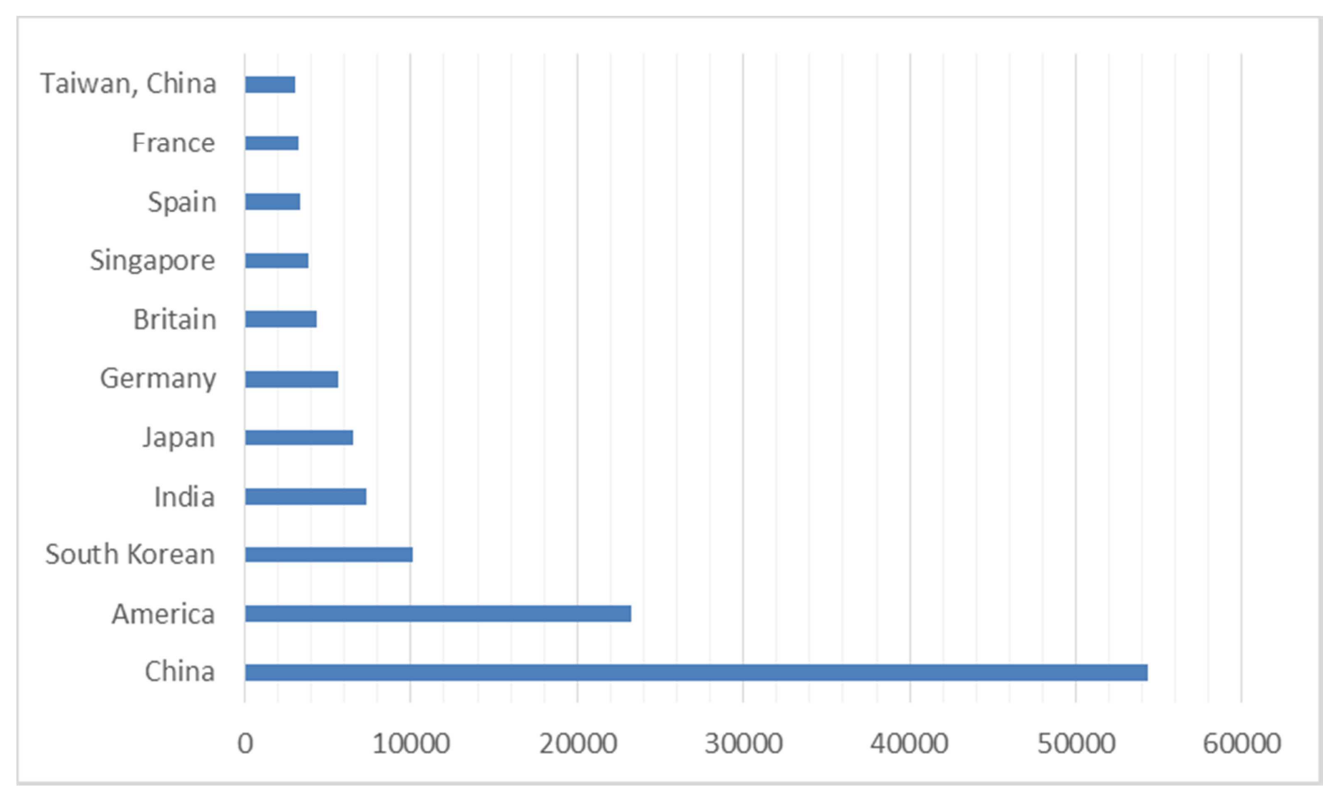

Figure 3. Quantity of graphene research papers published in major countries. 
Table 2. Support funds and agency statistics in the field of graphene.

\begin{tabular}{llll}
\hline NO & Scientific Research Institutions & Country & Amount \\
\hline 1 & China National Natural Science Fund & China & 37094 \\
2 & Fundamental Research Funds for the Central Universities & China & 5085 \\
3 & National Science Foundation, United States & United States & 4946 \\
4 & China national key basic research and development plan & China & 3277 \\
5 & China Postdoctoral Science Foundation funded project & China & 2006 \\
6 & A Project Funded by the Priority Academic Program Development of Jiang su Higher Education Institutions & China & 1572 \\
7 & National Program on Key Basic Research Project (973 Program) & China & 1308 \\
8 & Science Foundation of the Chinese Academy of Sciences & China & 1169 \\
9 & Program for New Century Excellent Talents in University & China & 1160 \\
10 & The natural science foundation of Jiangsu Province & China & 1102 \\
11 & MINISTRY OF SCIENCE AND TECHNOLOGY OF CHINA & China \\
12 & Engineering and Physical Sciences Research Council(EPSRC) & United Kingdom & 861 \\
13 & EUROPEAN UNION & European Union & 734 \\
14 & AUSTRALIAN RESEARCH COUNCIL & Australia & 702 \\
15 & RUSSIAN FOUNDATION FOR BASIC RESEARCH & Russia & 685 \\
16 & CHINA SCHOLARSHIP COUNCIL & China \\
17 & MINISTRY OF EDUCATION SCIENCE AND TECHNOLOGY & South's Korea & 663 \\
18 & DFG & Switzerland & 654 \\
19 & NATIONAL RESEARCH FOUNDATION OF KOREA NRF & South's Korea & 638 \\
20 & SPECIALIZED RESEARCH FUND FOR THE DOCTORAL PROGRAM OF HIGHER EDUCATION & China & 631 \\
\hline & & &
\end{tabular}

\subsection{Measure of Indicators}

The centrality of various countries in the graphene research network was studied. Centrality was used to measure the central position of a research institution or country in a network of scientific knowledge citations, or to measure the core position of a research topic in the field. As shown in
Figure 4, the large points in the graph indicate strong centrality and demonstrate that the papers were cited by more countries. The centrality and innovation of the United Kingdome and Germany were strong. Sigma was an indicator of the frontier. The United Kingdom was better at the frontier of research, and Germany was better at research centrality.

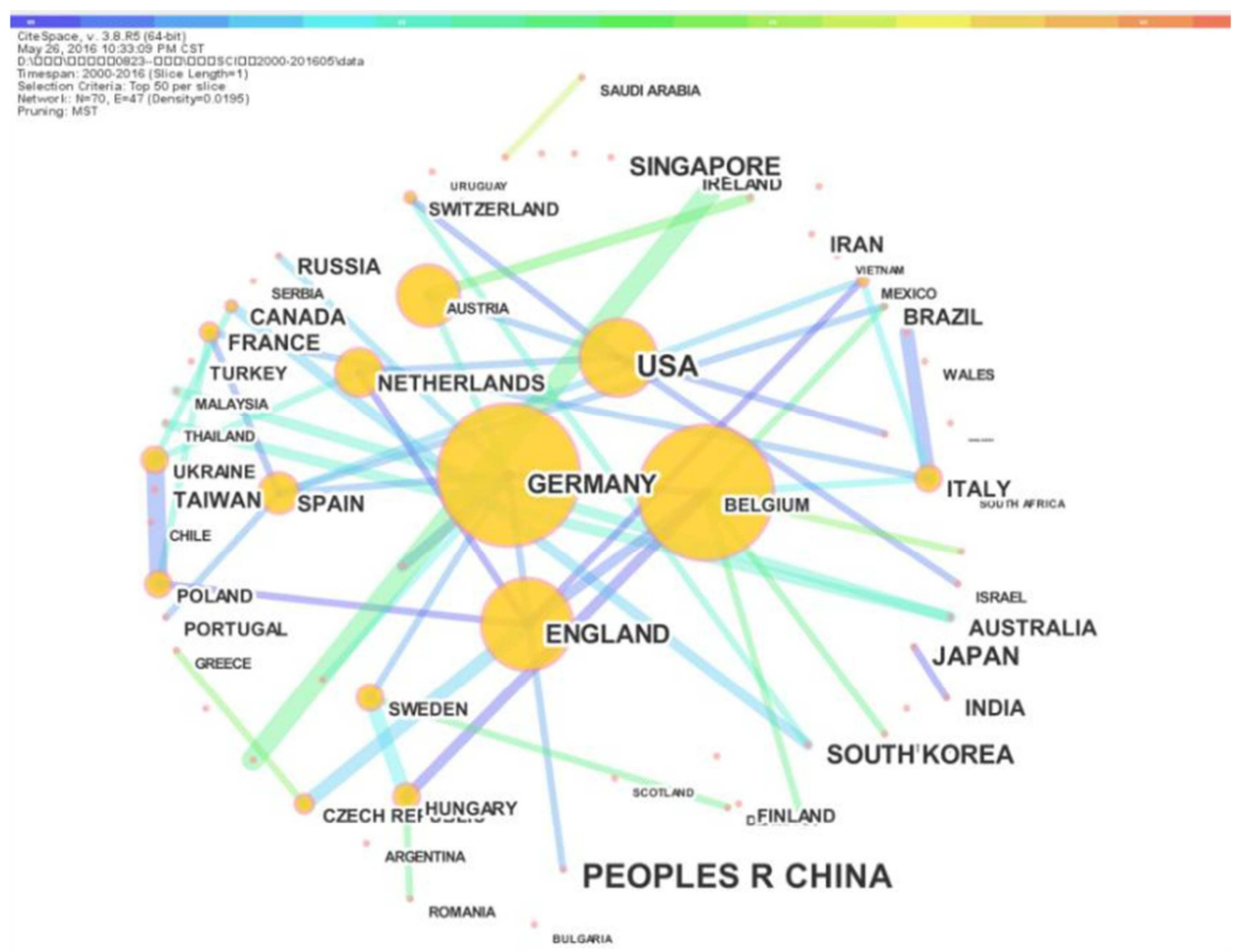

(The size of the country name indicates the number of times cited, and the size of the node represents the centrality of the network of scientific papers.).

Figure 4. The number and centrality of the papers in the field of graphene. 


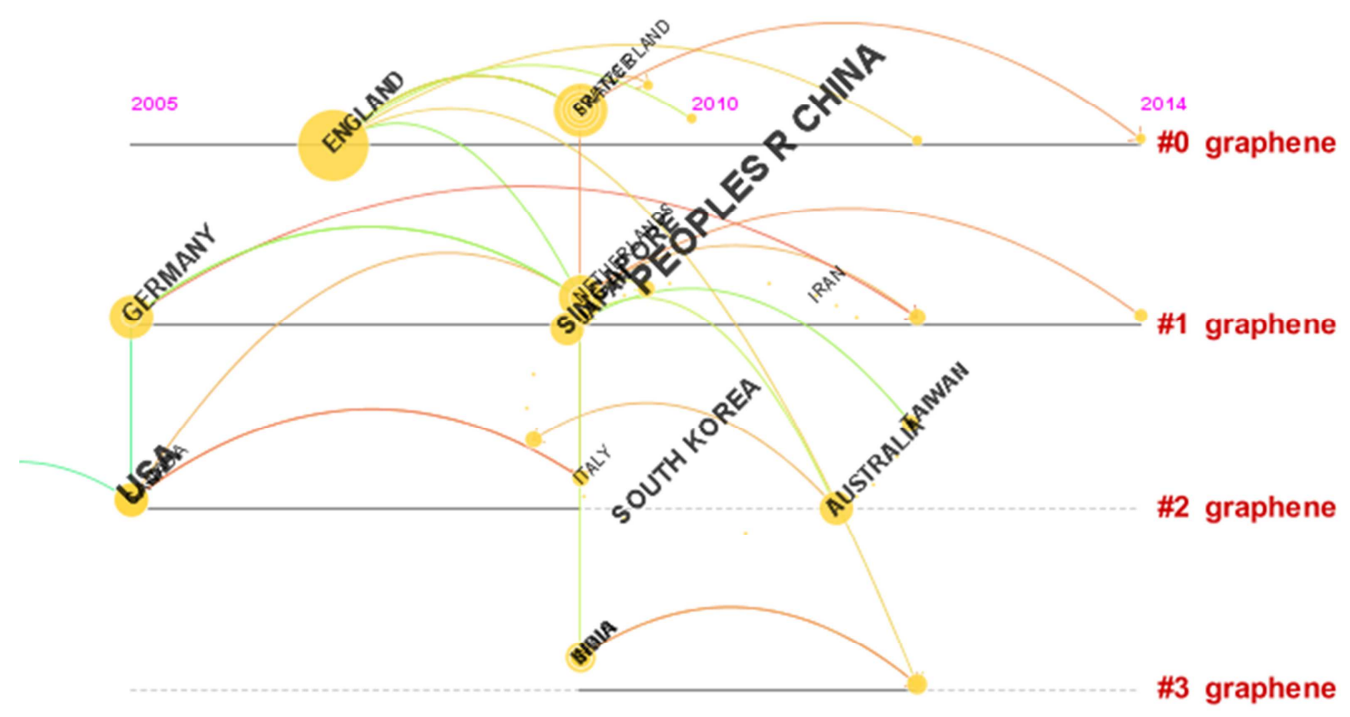

Figure 5. Main topics and temporal variation of graphene.

(0: battery electrode material, lithium ion battery; 1 : nanographite particle complex, active catalyst, fuel electrode; 2 . Multilayer graphene film, transparent electrode film, and transparent conductive electrode; 3: functional research, electronic performance, and electron transfer)

Judging from the chronological order, the United States and Germany conducted a large number of graphene studies around 2005. The United Kingdom launched a large number of graphene studies in 2007. China developed graphene research around 2009. Different countries had different research themes. The United Kingdom and Belgium mainly focused on battery electrode materials and lithium-ion batteries. Germany, China, and Singapore mainly focused on nanographite particle composites, active catalysts, and fuel electrodes. The United States, South Korea, and Australia mainly concentrated on multilayer graphene films, transparent electrode films, and transparent conductive electrodes. India mainly focused on functional research, electronic properties, and electron transfer, as shown in Figure 5.

\subsection{Comprehensive Ranking Evaluation}

The performance of scientific innovation indicators in various countries of graphene is shown in Table 3. China had an obvious advantage in the quantity of published papers, which ranked first in the world. The United Kingdom ranked first in the frontier of scientific research, and Germany was first in research centrality. According to the TOPSIS algorithm, the comprehensive evaluation value of the United States ranked first in the world. Germany, Britain, China, and Spain were in second to fifth place, respectively.

Table 3. Ranking of the major countries in graphene.

\begin{tabular}{llllll}
\hline Ranking & Country & Quantity & Centrality & Frontier & Comprehensive evaluation value $^{*}$ \\
\hline 1 & United States & 4902 & 0.1 & 2.09 & 0.632 \\
2 & Germany & 1163 & 0.19 & 2.12 & 0.568 \\
3 & United Kingdom & 691 & 0.12 & 3.07 & 0.471 \\
4 & China & 5693 & 0 & 1.00 & 0.435 \\
5 & Spain & 608 & 0.06 & 1.80 & 0.247 \\
6 & Japan & 1481 & 0 & 1.00 & 0.109 \\
7 & South Korea & 1340 & 0.01 & 1.00 & 0.102 \\
8 & France & 633 & 0.02 & 1.32 & 0.0928 \\
9 & Singapore & 791 & 0 & 1.00 & 0.0333 \\
10 & India & 611 & 0 & 1.00 & 0.0125 \\
\hline
\end{tabular}

By using the TOPSIS algorithm, the distance to the ideal solution was calculated and the comprehensive evaluation value was obtained.

We invited a number of graphene experts to consult on issues of field classification, key technologies, and scientific evaluation indicators. Experts evaluated the reasonableness of the evaluation. According to expert judgment, the ranking of competitiveness evaluation of the world's major countries in the field of graphene was recognized.

\section{Conclusions}

On the basis of this evaluation, the following conclusions was made:

(1) On the basis of the value of basic academic research, a model was constructed to evaluate national academic competitiveness in scientific research fields. The evaluation index system included the quantitative 
indicators, influence indicators, and hot spots and frontier indicators of academic research innovation.

(2) Frontier indicators were introduced to reflect the academic innovation ability of research fields by calculating the continuous attention of experts drawn to a certain achievement in the scientific field for a period of time.

(3) On the basis of the evaluation model of academic competitiveness in the scientific field formed in this paper, an empirical study in the field of graphene was conducted, which formed a basic evaluation of the academic competitiveness of graphene and a national ranking. Through a questionnaire survey and expert consultation, the evaluation method of this study was deemed, the indicators were reliable, and the evaluation results were recognized by experts

(4) Through the analysis of the scientific competitiveness of major countries in the world of graphene, the results showed that China has obvious advantages in the number of published papers and citations, which ranked first in the world. The United Kingdom ranked first in the frontier of scientific research, and Germany was the first in research centrality. Using the TOPSIS algorithm to comprehensively evaluate the academic innovation in the field of graphene, the United States achieved good results in quantity, centrality, and hot spots, and ranked first in the world. Germany, Britain, China, and Spain were ranked from second to fifth place, respectively.

(5) This article introduced centrality, hot spots and research frontiers as well as other indicators. Using these indicators and models, cutting-edge scientists and research teams that have made outstanding achievements in certain fields can be identified quickly, which can guide countries as they strengthen support for these scientists and seek to form research teams.

\section{Acknowledgements}

The authors acknowledge the financial support from the National Soft Science Research Program of China (Grant No.2013GXS6k208).

\section{References}

[1] Bai Chun Li. Earnestly implement the guidelines of the new period and strengthen the basic research work of our college Report on the work of the basic research conference of the Chinese Academy of Sciences, 2002 (6).

[2] Yu Jianbin. Zhang. Science and technology innovation has entered the new phase of "three runs coexist". People's daily, 2016-03-11 (02).

[3] Ren Zhengfei. HUAWEI is in an innovation dilemma that no one has guided. No one follows. "National Conference on science and technology innovation", 2016-06-02.

[4] leaf trabeculae. How to evaluate the performance of basic research. Science news, 2003, 6:39-40.
[5] Wan Yuling, Hou Xiaoxia. Discussion on the evaluation of basic research in China. Chinese Basic Science, 1999, (2-4):25-29.

[6] Shen Xin Yin. Reflections on some methods of performance evaluation of the National Science Foundation of the United States. China Science Foundation, 2001, 15 (5): 313-316

[7] Zhou Hongfang, Chen Wenxian, Zhang Lin. Preliminary study on performance evaluation of medical basic research projects. Chinese medical research management, 2002, 15 (3): 155-157.

[8] Shan Guang Liang, Zhao Aifang. Discussion on the performance evaluation system of basic researchers. Chinese medical research management, 2002,15 (4): 224-226

[9] Shaw. Methods and Revelations of scientific and technological assessment in developed countries. Influence of science on society, 2001 (4): 18-20.

[10] Xu F, Li X X, Meng W, et al. Ranking academic.

[11] Jiang Chunlin, Liu Zeyuan, Liang Yongxia, H index and G index: a new index for evaluating academic influence of journals. Library and information work, 2006, 50 (12): 63-65.

[12] Liu Yajuan, Wang Yan. Several indicators for basic research on Bibliometrics: paper, citation and journal impact factors. Scientific research management, 2000, 21 (1): 93-98.

[13] Freeman L C. A set of measures of centrality based on, 1977, 40 (1): Liu Jun. Introduction to social network analysis. Beijing: Social Science Literature Press.

[14] Liu Jun. Introduction to Social Network Analysis. Beijing: Social Science Literature Publishing House, 2004.134-137.

[15] Zhang Xuan, Wang Guoshun, Bi Xiaoping. Influence of network centrality and knowledge innovation capability on innovation performance. Economic problems, 2013 (8): 92-96.

[16] Zheng Dengpan, Party Xinghua. Research on the measurement of innovation center's centrality in technological innovation network based on social network analysis. Journal of systems management, 2010, 19 (8): 415-419.

[17] Luan Chun Ju. Application of network centrality index in technology measurement, scientific and technological progress and countermeasures, 2013, 30 (2): 10-13.

[18] Chen C M. CiteSpace II: Detecting and visualizing emerging trends and transient, 2006, 57 (3).

[19] Zhang Jing. Research on agricultural science and technology innovation ability and efficiency in China. Yangling: Northwest Agriculture and Forestry University, 2013.

[20] Wang Zeyu, Liu Fengzhao. Analysis of the coordination between marine science and technology innovation capability and marine economic development in China. Science and technology management, 2011.32 (5): 42-47.

[21] Gao Feng, Dabg Yaru. Construction of civil aviation science and technology innovation evaluation index system. Research management research. 2005, 25 (8): 35-37.

[22] Zhou Jian, Chen Jie. Construction of evaluation index system for two integration of manufacturing enterprises. Computer integrated manufacturing system. 2013,19 (9): 2251-2263.

[23] Wei Jiang, Gou Li, Zhou Min Fei. Analysis of performance indicators of basic research. Journal of Nanjing University of Science and Technology, 2008,21 (4): 64-69. 
[24] Xu Fang, Liu Wenbin, Li Xiaoxuan. Methods and implications of FER scientific research impact assessment in UK. Science and technology management, 2014,35 (7): 9-15.

[25] Song Liping. REF and the trend of scientific research evaluation. Library and information work, 2011 (22): 60-63100.

[26] Du Debin, Zhang Ren Kai, Li Pengfei. The REF evaluation system of British universities and its enlightenment, Chinese University Science and technology, 2014, 03: 36-38.
[27] Bonacich P. Factoring and weighting approaches to status scores and scores $1972 \mathrm{P}$.

[28] Zhang Lihua, Zhang Zhiqiang. Advances in frontiers migration research. Library and information services, 2014, 58 (3): 5-12, 19.

[29] Jon K. Bursty and Hierarchical Structure in Streams. Data Mining \& Data, 2003, 7 (4). 